\title{
From Geometric Quantization to Conformal Field Theory
}

\author{
A. Alekseev and S. Shatashvili \\ Leningrad Steklov Mathematical Institute, Fontanka 27, SU-191011 Leningrad, USSR
}

\begin{abstract}
Investigation of $2 d$ conformal field theory in terms of geometric quantization is given. We quantize the so-called model space of the compact Lie group, Virasoro group and Kac-Moody group. In particular, we give a geometrical interpretation of the Virasoro discrete series and explain that this type of geometric quantization reproduces the chiral part of CFT (minimal models, $2 d$-gravity, WZNW theory). In the appendix we discuss the relation between classical (constant) $r$-matrices and this geometrical approach.
\end{abstract}

\section{Introduction}

In this paper we continue an investigation of $2 d$ conformal field theories in terms of geometric quantization (see [1-3]). As demonstrated in our previous papers, the standard geometric quantization method [4] can be reformulated in terms of the path integral approach. In [2] the correspondence between the coadjoint orbit and the irreducible representation of compact Lie groups was explicitly realized by means of the functional integral. More precisely, we constructed in [2] a quantum mechanical system, such that the path integral with boundary conditions gives matrix coefficients of the corresponding irreducible representation. The action functional of this system is defined by the canonical symplectic structure $\Omega$ on the given coadjoint orbit and a Hamiltonian $H(X)$, which is a function on the orbit: $S=\int d^{-1} \Omega-\int H(X) d t$; this action is a functional of trajectories on the orbit. Later in [3] using the same rules, we described quantum field theory on the coadjoint orbit of infinite dimensional Lie groups (Virasoro, Kac-Moody) and the properties of the corresponding action functional investigated. In particular, we have shown that for the Virasoro group the geometrical action, written in terms of group variables $F(x) \in \operatorname{diff} S^{1}$ differs from the action in $2 d$ gravity [5] by the extra term $\int b_{0} \dot{F} F^{\prime} d x d t$, where the number $b_{0}$ parametrizes generic coadjoint orbits. (A similar statement is true also for Kac-Moody group and WZNW model.) In the language of geometric quantization the appearance of $S L(2, \mathbf{R})$ current algebra in $2 d$ gravity is the consequence of symplectic geometry, and as it was shown in [3] Virasoro 
geometrical action can be obtained from the $S L(2, \mathbf{R})$ Kac-Moody one by the Lagrangian version of the Drinfeld-Sokolov Hamiltonian reduction (see also [6]).

Here we will give a slightly different type of geometric construction in which all representations of the group are considered simultaneously and on the same footing. More precisely, using the path integral approach, we will quantize the socalled model space, i.e. such space that its quantization yields all representations of the group with multiplicity one. This space is larger than the coadjoint orbit (roughly speaking, it contains an extra variable which parametrizes the orbits and the conjugate moment). The corresponding Hilbert space splits into the direct sum of all irreducible representations. Model spaces for compact semisimple Lie groups have been studied earlier in [7]. (So far, we have been unable to compare our construction with those in [7].) We believe that the study of model space provides a more natural language for geometric quantization, especially in the infinite dimensional case. It seems likely that for Virasoro and Kac-Moody groups the quantization of individual orbits gives rise only to Verma modules. By contrast, quantization of the model space gives rise (see Sects. 3 and 4 below) to their irreducible quotients. Moreover, this type of geometric quantization, as will be explained in Sects. 3 and 4 reproduces the chiral part in conformal field theories and therefore it is also a natural language for them. Physically, this construction means that path integrals are averaged over the set of all orbits. More exactly, the parameter which lables the Virasoro coadjoint orbit becomes a quantummechanical dynamical variable. The geometrical action, defined in our previous papers (more accurately its trivial generalization) plays in this construction a crucial role. It defines the symplectic structure on the model space. Using the technique developed in $[1,2,3]$ it is possible to introduce the "Darboux" variables for this symplectic form both in the finite dimensional case and in the Virasoro case (in the Kac-Moody group case - only for the quantum-mechanical part). As a result the path integral reduces to a sum over blocks, where each of them is also path integral, but over a special set of orbits. For the case of the Kac-Moody group this sum is finite and each block corresponds to an integrable representation of the group (this construction gives precisely a chiral part in WZNW model). For the case of the Virasoro group with $C_{q}<1$ we also get a finite sum, with each block corresponding to an irreducible representation of the group (for exact an statement, see below in Sect. 3) and the central charge also quantized $C_{q}=1$ $-6 \frac{(p-q)^{2}}{p q}$; the sum is over Virasoro discrete series. These properties are similar to those for the finite dimensional case.

This paper is organized as follows. In Sect. 2 we consider the quantization of the cotangent bundle $T^{*} G$ and model space for the compact Lie groups. In Sects. 3 and 4 using the path integral approach for the quantization of model space for Virasoro and Kac-Moody groups correspondingly we reproduce the finite sums in RCFT. In the appendix we discuss an interesting question on the relation between the geometrical actions and classical $r$-matrices (without the spectral parameter). Our main observation is that the geometrical action defines a nondegenerate symplectic structure on the group (in the infinite-dimensional case) where corresponding Poisson brackets are of the $r$-matrix nature. It means that there is a relation between RCFT and quantum groups just on the classical level. 
We think that this observation together with the geometrical point of view on the conformal field theory can explain in the future the appearance of quantum groups in RCFT. We will return to this subject in future publications.

\section{Quantization of the $T^{*} G$ and the Model Space}

The most natural symplectic manifold, related to the group is $T^{*} G$ - the cotangent bundle of the group $G$ (in this section we will consider a compact Lie group $G$ ). By using Hamiltonian reduction we replace it with a smaller space, which will be called the model space. The quantization of this space yields all irreducible unitary representations of the group with multiplicity one. The quantization of the model space is performed via the path integral method. For concreteness we shall consider below only the case $G=S U(n)$. A generalization to arbitrary simple groups is straightforward (cf. [2]).

The canonical symplectic 2 -form on $T^{*} G$ is defined by

$$
\Omega=\frac{1}{2}\left(\operatorname{tr} d X d g \cdot g^{-1}+\operatorname{tr} X\left(d g \cdot g^{-1}\right)^{2}\right),
$$

where $g \in G, X \in \mathscr{G}^{*}$ is the right-invariant moment, $\mathscr{G}^{*}$ is the space dual to the corresponding Lie algebra $\mathscr{G}$. As in [1-3] we define the geometric action on $T^{*} G$ as a functional of trajectories on the $T^{*} G$

here $d \alpha=\Omega$.

$$
S=\frac{1}{2} \int \operatorname{tr} X d g \cdot g^{-1}=\int \alpha
$$

This geometrical action possesses two symmetries:

$$
\begin{gathered}
X(t) \rightarrow X(t), \quad g(t) \rightarrow g(t) h_{R}, \\
X(t) \rightarrow h_{L} X(t) h_{L}^{-1}, \quad g(t) \rightarrow h_{L} g(t) .
\end{gathered}
$$

Here $h_{R}$ and $h_{L}$ are constant matrices from $G$. Using the parametrization

$$
X=f X_{0} f^{-1}, \quad f \in G,
$$

where $X_{0}$ is a diagonal matrix we get

$$
S=\frac{1}{2} \int \operatorname{tr}\left[X_{0} d\left(f^{-1} g\right)\left(f^{-1} g\right)^{-1}+X_{0} f^{-1} d f\right],
$$

and in the notation $\tilde{g}=f^{-1} g$ the action acquires the form

$$
S=\frac{1}{2} \int \operatorname{tr}\left[X_{0} d \tilde{g} \cdot \tilde{g}^{-1}+X_{0} f^{-1} d f\right] .
$$

$f$ is defined modulo transformation $f \rightarrow f h$, with $h \in H ; H$ is the stationary subgroup of $X_{0}$, i.e. $h$ is a diagonal matrix from the Cartan subgroup of $G$.

In new variables the global symmetries (3) are expressed by

$$
\begin{array}{lll}
\tilde{g}(t) \rightarrow \tilde{g}(t) h_{R}, & f(t) \rightarrow f(t), & X_{0}(t) \rightarrow X_{0}(t), \\
\tilde{g}(t) \rightarrow \tilde{g}(t), & f(t) \rightarrow h_{L} f(t), & X_{0}(t) \rightarrow X_{0}(t) .
\end{array}
$$

It means that $\tilde{g}(f)$ possesses only right (left) symmetry.

There is also an additional local symmetry in the action

$$
X_{0}(t) \rightarrow g_{0}(t) X_{0}(t) g_{0}^{-1}(t) ; \quad f(t) \rightarrow f(t) g_{0}^{-1}(t) ; \quad \tilde{g}(t) \rightarrow g_{0}(t) \tilde{g}(t),
$$


where $g_{0}(t)$ belongs to the stabilizer of the Cartan subalgebra, i.e. $g_{0}$ is such an element of the group that $\bar{X}_{0}=g_{0} X_{0} g_{0}^{-1}$ is again diagonal. The eigenvalues of the matrices $X_{0}$ and $\tilde{X}_{0}$ coincide and both are diagonal, it means that the subgroup of $G$ formed by these transformations contains a component, which acts on the eigenvalues of $X_{0}$ as a permutation, and therefore this action can be identified with the corresponding Weyl group.

Let $e^{i}, i=1, \ldots, n$ be an orthonormal basis in the $n$-dimensional complex vector space, in which $X_{0}$ is a diagonal with eigenvalues $2 m_{1}^{0}\left(t_{i}\right), \ldots, 2 m_{n i}^{0} ; \sum_{1}^{n} m_{i}^{(0)}=0$. Using the symmetry with respect to the permutations, discussed above, we may impose the restriction $m_{1}^{0} \geqq m_{2}^{0} \geqq \ldots \geqq m_{n}^{0}$, which is a fundamental domain of the Weyl group, the Weyl chamber. Then it can be shown (see [2]) that each term in (4) acquires the form

$$
\frac{1}{2} \operatorname{tr} X_{0} d \tilde{g} \tilde{g}^{-1}=\frac{i}{2} \sum_{1}^{n} m_{i}^{0}(t)\left[\left(d a_{i}, a_{i}\right)-\left(a_{i} d a_{i}\right)\right],
$$

where $a^{i}(t)=g(t) e^{i}$. The new vectors, $a^{i}$, are also orthonormal. Let us parametrize the last component of all these vectors by the angles $\varphi_{i}^{0}$ :

$$
a_{n}^{i}=e^{i \varphi_{i}^{0}} \tilde{a}_{n}^{i}
$$

where $\tilde{a}_{n}^{i}-$ are real: $\operatorname{Im} \tilde{a}_{n}^{i}=0$. Then we get

$$
\frac{1}{2} \operatorname{tr} X_{0} d \tilde{g} \tilde{g}^{-1}=\sum_{1}^{n} m_{i}^{0} d \varphi_{i}^{0}+\frac{i}{2} \sum_{1}^{n} m_{i}^{0}\left[\left(d \tilde{a}_{i}, \tilde{a}_{i}\right)-\left(\tilde{a}_{i}, d \tilde{a}_{i}\right)\right] .
$$

Using the construction, developed in [2], for the second term in (9) we obtain the expression

$$
\frac{1}{2} \operatorname{tr} X_{0} d \tilde{g} \tilde{g}^{-1}=\sum_{1}^{n} m_{i}^{0} d \varphi_{i}^{0}+\sum_{i, k} m_{i}^{(k)} d \varphi_{i}^{(k)},
$$

where $\varphi_{i}^{0}, \varphi_{i}^{(k)}$ are angles, $0 \leqq \varphi_{i}^{(k)} \leqq 2 \pi$ and the variables $m_{i}^{(k)}$ form a Polyhedron $\Pi_{m}$ of the dimension $\frac{n^{2}-n}{2}=\frac{\operatorname{dim} G-\operatorname{rank} G}{2}$,

$$
\prod_{m}^{\frac{n^{2}-n}{2}}: m_{i}^{(k-1)} \geqq m_{i}^{(k)} \geqq m_{i+1}^{(k-1)},
$$

which is the classical analog of the Gelfand-Zetlin basis.

The same construction can be applied to the second term in (4) and we finally get

$$
S=\int\left(\sum_{1}^{n} m_{i}^{0}\left(d \varphi_{i}^{0}+d \psi_{i}^{0}\right)+\sum_{i, k} m_{i}^{(k)} d \varphi_{i}^{(k)}+\sum_{i, k} n_{i}^{(k)} d \psi_{i}^{(k)}\right),
$$

where $n_{i}^{(k)}$ form the polyhedron $\Pi_{n}$, defined by the "highest" weight $\left(-m_{n}^{0}, \ldots,-m_{1}^{0}\right)$ and $\psi_{i}^{(k)}$ are the corresponding angle variables.

Now we must remember that $f$ was defined module transformations $f \rightarrow f h: h \in H$ and therefore we can choose $\psi_{i}^{(0)}=0, i=i, \ldots, n-1$. Conditions $\operatorname{det} \tilde{g}=1$ and $\operatorname{det} f=1$ in terms of $m, \varphi$ variables can be written as

$$
\sum_{i}^{n} \varphi_{i}^{(0)}+\sum_{i, k} \varphi_{i}^{(k)}=0, \quad \psi_{n}^{(0)}+\sum_{i, k} \psi_{i}^{(k)}=0
$$


This finally yields the following expression for the action:

$$
S=\int\left(\sum_{1}^{r} \Delta_{i} d \varphi_{i}^{(0)}+\sum_{i, k} \Delta_{i}^{(k)} d \varphi_{i}^{(k)}+\sum_{i, k} \bar{\Delta}_{i}^{(k)} d \psi_{i}^{(k)}\right),
$$

where $\quad \Delta_{i}=m_{i}^{(0)}-m_{n}^{(0)}, \quad \Delta_{i}^{(k)}=m_{i}^{(k)}-m_{n}^{(0)}, \quad$ and $\quad \bar{\Delta}_{i}^{(k)}=n_{i}^{(k)}+m_{n}^{(0)}$; $\Delta_{1} \geqq \Delta_{2} \geqq \ldots \geqq \Delta_{n-1} \geqq 0 ; \Delta \in \Pi$ and the corresponding regions for $\Delta_{i}^{(n)}$ and $\bar{\Delta}_{i}^{(k)}$ we denote by $\Pi_{\Delta}, \Pi_{\bar{\Delta}}$.

Thus we constructed the "Darboux" variables, and so we can easily reduce this system to a smaller one. The first is the reduction over the constraints $\psi_{i}^{(k)}=$ const. These constraints kill the field $f$. The resulting system is the so-called model space. The corresponding Hilbert space will be $\mathscr{H}=\bigoplus_{i} \mathscr{H}_{i}$, where $\mathscr{H}_{i}$ are all irreducible representations of the group. If we impose an extra condition $m_{i}^{(0)}=M_{i}=$ const we will come back to the orbit, corresponding to the point $X_{0}=\operatorname{diag}\left(M_{i}\right)$.

Let us now consider the path integral quantization of the system under consideration. Following [2] the Hamiltonian is chosen to be a linear combination of Cartan elements $H_{i}: H=\alpha_{i} H_{i}(m)+\beta_{i} H_{i}(n)$. In the $S U(n)$ case Cartan elements $H_{i}$ are Gelfand-Zetlin parametrization and are given by the sum $H_{i}=\sum_{l} m_{i}^{(k)}$ over the rows $i$ of the classical Gelfand-Zetlin table. Thus

$$
\widetilde{S}=\int\left(\sum_{1}^{r} \Delta_{i} d \varphi_{i}^{(0)}+\sum_{i, k} \Delta_{i}^{(k)}+\sum_{i, k} \bar{\Delta}_{i}^{k} d \psi_{i}^{(k)}\right)-\int\left(\alpha_{i} H_{i}(m)+\beta_{i} H_{i}(n)\right) d t .
$$

As in the case of the coadjoint orbit (see [2]) the path integral

$$
G\left(\varphi^{0^{\prime \prime}}, \varphi^{\prime \prime}, \psi^{\prime \prime} \mid \varphi^{0^{\prime}}, \varphi^{\prime}, \psi^{\prime}\right)=\int d \Delta_{i} d \varphi_{i}^{0} d \Delta_{i}^{(k)} d \bar{\Delta}_{i}^{(k)} d \varphi_{i}^{(k)} d \psi_{i}^{(k)} e^{i \tilde{S}}
$$

with the boundary conditions: $\varphi_{i}^{(0)}(0)=\varphi_{i}^{(0)}, \varphi_{i}^{(k)}(0)=\varphi_{i}^{(k)}, \psi_{i}^{(k)}(0)=\psi_{i}^{(k)^{\prime}}$, and

$$
\varphi^{0}(T), \varphi(T), \psi(T)=\varphi^{(0)^{\prime \prime}}, \varphi^{\prime \prime}, \psi^{\prime \prime}
$$

can be easily calculated and we get

$$
\begin{aligned}
G\left(\varphi^{0^{\prime \prime}}, \varphi^{\prime \prime}, \psi^{\prime \prime} \mid \varphi^{0^{\prime}}, \varphi^{\prime}, \psi^{\prime}\right)= & \sum_{\Delta \in \Pi \mathbf{z}} \exp \left(i \sum_{i} \Delta_{i}\left(\varphi^{(0)^{\prime \prime}}-\varphi^{(0)^{\prime}}\right)\right) \\
& \times \sum_{\Delta_{i}^{(k)} \in \Pi_{\Delta} \mathbf{z}} \exp \left(i \sum_{i, k} \Delta_{i}^{(k)}\left(\varphi_{i}^{(k)^{\prime \prime}}-\varphi_{i}^{(k)^{\prime}}\right)-i H(m) T\right) \\
& \times \sum_{\bar{\Delta}_{i}^{(k)} \in I_{\bar{\Delta}} \mathbf{z}} \exp \left(i \sum_{i, K} \bar{\Delta}_{i}^{(k)}\left(\psi_{i}^{(k)^{\prime \prime}}-\psi_{i}^{(k)^{\prime}}\right)-i H(n) T\right),
\end{aligned}
$$

where $\Pi_{\Delta, \mathbf{z}}$ is integer valued Gelfand-Zetlin table. From this we obtain that the Hilbert space is direct sum $\mathscr{H}=\bigoplus_{i} \mathscr{H}_{i}{ }^{L} \otimes \mathscr{H}_{i}{ }^{R}$, where $\mathscr{H}_{i}{ }^{L}$ and $\mathscr{H}_{i}{ }^{R}$ are irreducible representations of the group where left and right translations are acting. This decomposition of $L_{2}(G)$ is well known. [Character can be obtained from (15) if we put $\varphi^{\prime}=\varphi^{\prime \prime}, \psi^{\prime}=\psi^{\prime \prime}$ and integrate over $\varphi, \psi$. $]$ Remark that this situation is quite similar to that in CFT. Maybe the study of the same construction for the infinitedimensional Lie groups (Virasoro, Kac-Moody) and their cosets, or DrinfeldSokolov reductions will help us to understand the geometrical nature of CFT. 
In the case of the model space we have

$$
\begin{aligned}
G\left(\varphi^{\prime}, \varphi^{\prime \prime}\right)= & \int d \Delta_{i} d \varphi_{i}^{0} d \Delta_{i}^{(k)} d \varphi_{i}^{(k)} \exp \tilde{S}\left(\Delta_{i}, \varphi_{i}^{0}, \Delta_{i}^{(k)}, \varphi_{i}^{(k)}\right) \\
= & \sum_{\Delta \in \Pi_{\Delta, \mathbf{z}}} \exp \left(i \sum_{i} \Delta_{i}\left(\varphi_{i}^{(0)^{\prime \prime}}-\varphi_{i}^{(0)^{\prime}}\right)\right) \\
& \times \sum_{\Delta_{i} \in \Pi_{\Delta, \mathbf{z}}} \exp \left(i \sum_{i, k} \Delta_{i}^{(k)}\left(\varphi_{i}^{(k)^{\prime \prime}}-\varphi_{i}^{(k)^{\prime}}\right)-i H(m) T\right) .
\end{aligned}
$$

and here $\mathscr{H}=\bigoplus_{i} \mathscr{H}_{i}$.

\section{Finite Sums in the RCFT; Virasoro Group}

First we will realize the above program for the Virasoro group. The main point we are going to explain is the geometrical interpretation of the $C_{q}<1$ discrete series. Here additional difficulties are present as a result of the infinite-dimensionality of the group and we will deal with 2-dimensional field theory instead of quantum mechanics. On the other hand the dimension of the space quantization which is responsible for the singling out the degenerate representations, is finite. This is the consequence of the finiteness of the rank.

The geometrical action for the Virasoro group was constructed in [3],

$$
A(F)=\int d x d t\left[-b_{0} \dot{F} F^{\prime}+\frac{C}{48 \pi} \frac{\dot{F}}{F^{\prime}}\left(\frac{F^{\prime \prime \prime}}{F^{\prime}}-2\left(\frac{F^{\prime \prime}}{F^{\prime}}\right)^{2}\right)\right],
$$

where $x \in S^{1}, F(x) \in \operatorname{diff} S^{1}, F(x+2 \pi)=F(x)+2 \pi$ and $F^{\prime}(x)>0$. In [3] also a natural measure for the path integral was given:

$$
\mu(F)=\prod \frac{d F}{F^{\prime}} .
$$

$b_{0}$ in (17) is the number which parametrizes the coadjoint orbits (it corresponds to classical "highest weight") and $C$ is the classical central charge.

In the future, for the construction of the model space we need a different form of the action (17). First we will introduce a new field $f=\exp \mu F$, where $\mu=\sqrt{\frac{48 \pi b_{0}}{C}}$
and the action acquires the form

$$
A(f)=\frac{C}{48 \pi} \int d x d t \frac{\dot{f}}{f^{\prime}}\left[\frac{f^{\prime \prime \prime}}{f^{\prime}}-2\left(\frac{f^{\prime \prime}}{f^{\prime}}\right)^{2}\right] .
$$

Now for the $f$ we have a boundary condition

$$
f(2 \pi)=e^{2 \pi \mu} f(0) .
$$

It is more convenient to use the parameter $\mu$ instead of the $b_{0}$. These are two different regions in the parameter space: 1) $b_{0} / C>0$, i.e. $\mu$ is real, and 2) $b_{0} / C>0$, i.e. $\mu$ is imaginary $\mu=i \tilde{\mu}$ with real $\tilde{\mu}$. In new variables the measure in the path integral has the same form $\mu(f)=\prod \frac{d f}{f^{\prime}}$. For the real $\mu$ the property $f^{\prime}=e^{\mu F} \mu F^{\prime}>0$ allows us 
to introduce one more change of variables

$$
f^{\prime}=e^{\varphi} \text {. }
$$

We can do the same for the imaginary $\mu$, but now $f^{\prime}=\exp i \varphi$, where $i \varphi=i \tilde{\mu} F^{\prime}$ $+\ln i \tilde{\mu} F$ and we get a nontrivial situation with complex $\varphi$. As follows from the boundary condition (20) $\varphi(x, t)$ can be expanded as

$$
\begin{array}{ll}
\varphi(x, t)=\varphi_{0}(t)+\mu x+\tilde{\varphi}(x, t) ; & b_{0} / C>0, \\
\varphi(x, t)=\varphi_{0}(t)+\tilde{\mu} x+\tilde{\varphi}(x, t) ; & b_{0} / C<0,
\end{array}
$$

where in both cases $\tilde{\varphi}$ is a periodic function, but in the first case it is a real field and it is complex for $b_{0} / C<0 . \varphi_{0}(t), \mu$, and $\bar{\mu}$ are real. As it follows from the definition of $\varphi$ for the $b_{0} / C<0, \tilde{\mu}$, and $\varphi_{0}$ are defined modulo integers

$$
\begin{aligned}
& \varphi_{0} \sim \varphi_{0}+2 \pi n, \quad m, n \in \mathbb{Z}, \\
& \tilde{\mu} \sim \tilde{\mu}+m .
\end{aligned}
$$

Let us first consider a more simple situation, when $\mu$ is real. In $\varphi$ variables the classical action acquires the form

$$
A(\varphi)=-\frac{C}{48 \pi} \int d x d t \dot{\varphi} \varphi^{\prime} .
$$

But there is an anomaly in the measure, which corresponds to the nontrivial Jacobian $J=\operatorname{det} e^{-\varphi} \partial e^{\varphi}$ :

$$
\prod d \varphi=J \mu(f) \text {. }
$$

(A similar Jacobian appears in the bosonization procuedure for the WZNW model [8] and it is possible to use the Lagrangian version of the Drinfeld-Sokolov reduction [3] from the bosonized $S L(2, \mathbf{R})$ WZNW theory to define the present model.) Including this anomaly into the path integral we get the following expression for the effective Lagrangian:

$$
L_{\mathrm{eff}}=\frac{1}{8 \pi}\left[-\left(\frac{C}{6}+2\right) \dot{\varphi} \varphi^{\prime}+2 R \varphi\right],
$$

where $R$ is the curvature of the external 2-dimensional metric on the world sheet (it is necessary to introduce the external metric in the regularization of the determinant). We must take into account this curvature term when defining the stress-energy tensor; in all other places in this paper we can put it equal to zero, i.e. we can choose the flat external metric. Now the measure in the functional integral is a free measure: $\mu(\varphi)=\prod d \varphi$. (Let us note, that when we consider a fixed orbit, i.e. the value of the parameter $\mu$ is fixed, $\varphi$ is not a free field, because of the constraint $\oint e^{\varphi}=\exp 2 \pi \mu-1=$ const, which must be included into the path integral; this constraint is equivalent to the screening operator in the operator language, see [8]).

Under the parametrization $x \rightarrow x+\varepsilon(x), f$ behaves as a scalar field: $\delta f=\varepsilon f^{\prime}$. It means that $\varphi$ is not a usual scalar field, but

$$
\delta \varphi=\varepsilon \varphi^{\prime}+\varepsilon^{\prime} .
$$


Using this transformation, we can define the generator of the reparametrization, i.e. energy-momentum tensor

$$
\delta A_{\text {eff }}=\int \varepsilon \dot{T} d x d t
$$

and

$$
T=\frac{1}{8 \pi}\left[\left(\frac{C}{6}+2\right) \varphi^{\prime 2}-2\left(\frac{C}{6}+2\right) \varphi^{\prime \prime}-2 \varphi^{\prime \prime}\right]
$$

In the notation $\alpha^{2}=\frac{C}{6}+2, \phi=\alpha \varphi$ we get

$$
L_{\mathrm{eff}}=\frac{1}{8 \pi}\left[-\dot{\phi} \phi^{\prime}+\frac{2}{\alpha} R \phi\right], \quad T=\frac{1}{8 \pi}\left[\phi^{\prime 2}-2\left(\alpha+\frac{1}{\alpha}\right) \phi^{\prime \prime}\right] .
$$

Thus the central charge of the Virasoro algebra is equal to

$$
C_{q}=1+6\left(\alpha+\frac{1}{\alpha}\right)^{2}
$$

and $C_{q}>25$ for real $\alpha$.

Let us now consider the structure of the model space for the Virasoro group, corresponding to the Lagrangian (29), i.e. for the case $b_{0} / C>0$. Now $\mu$ also becomes a dynamical variable and

$$
A_{\text {eff }}=-\frac{\alpha^{2}}{2} \int d t \mu \dot{\varphi}_{0}+A_{\text {eff }}(\tilde{\varphi}) .
$$

The coefficient in the quantum mechanical term in (31) plays an important role in our further construction and therefore it must be computed from the starting action (17). (The difficulties appear during the change of variables we used before, because they were done for the constant $\mu$; the terms of interest are total derivatives and it is easy to forget them.) The first term in (31) comes from the $\Delta A_{c e}$ :

$$
\Delta A_{c e}=-\frac{C}{48 \pi} \int d x d t \mu^{2} \dot{F} F^{\prime},
$$

with $F=F_{0}+x+$ (period terms). From a comparison with (21) we get $F=\frac{\varphi_{0}}{\mu}+x+\ldots$ and therefore we have (up to the total derivative)

$$
\Delta A_{c e}=-\frac{C}{12} \int d t \mu \dot{\varphi}_{0}+\ldots,
$$

where terms ... don't contain $\mu$. As demonstrated before there is a renormalization $C \rightarrow 6 \alpha^{2}$ after quantization, and finally

$$
\Delta A_{q}=-\frac{\alpha^{2}}{2} \int d t \mu \dot{\varphi}_{0}
$$

This is the coefficient which was included in (31).

Now we have a pair of conjugate variables $\varphi_{0} \in(-\infty, \infty)$ and $\mu \in(0, \infty)$. The corresponding phase space is non-compact. Each value of $\mu=\mu_{0}$ (i.e. fixed coadjoint orbit) fixes a representation of the Virasoro group. Thus, the spectrum of 
the representations is continuous. The character of the given representation can be written as a path integral in the same way as for the compact groups. To this we must introduce the Hamiltonian $H=\int T(x, t) d x$, which is a Cartan element of the Virasoro algebra and calculate the path integral

$$
\int[d \varphi] \exp \left\{\left[A_{\mathrm{eff}}+\int_{0}^{T}\left(H-\frac{1}{24}\right) d t\right]\right\}=I\left(\mu^{\prime}, \mu^{\prime \prime} \mid T\right),
$$

with the boundary conditions $\tilde{\varphi}(0, x)=\tilde{\varphi}(T, x)$ and $\mu(0)=\mu^{\prime}, \mu(T)=\mu^{\prime \prime}$. It is easy to see that the path integral acquires the form

$$
I\left(\mu^{\prime}, \mu^{\prime \prime} \mid T\right)=\chi_{\mu^{\prime}}(T) \delta\left(\mu^{\prime}-\mu^{\prime \prime}\right),
$$

where $\chi_{\mu^{\prime}}(T)$ coincides with the character of the irreducible representation of the Virasoro group with the central charge $C_{q}>25$ and the highest weight $h=\frac{\alpha^{2} \mu^{2}}{4}$,

$$
\chi_{\mu}(T)=\frac{e^{2 \pi i h T}}{\eta(T)} .
$$

$\eta(T)$ - is a Dedekind eta function $\eta(T)=\exp \frac{i \pi T}{12} \prod_{n=1}^{\infty}\left(1-e^{2 \pi i n T}\right)$. Here we change $T$ by $2 \pi T$ to obtain the answer in the traditional form. (Our Hamiltonian can be written as

$$
H=\alpha^{2}\left[\frac{\mu^{2}}{4}+\frac{1}{2} \sum_{n \geqq 1} a_{n} a_{-n}\right],
$$

where $\varphi^{\prime}(x, t)=\mu(t)+\sum_{n \neq 0} a_{n}(t) e^{i n x}$, and it differs from the $L_{0}$ in the standard definition of the character

$$
\chi(q)=\operatorname{tr} q^{L_{0}-\frac{C}{24}}
$$

by shifting $H=L_{0}-\frac{C_{q}-1}{24}$. The term $(1-\exp 2 \pi i n T)$ comes from the integral over nonzero modes $a_{n}, a_{-n}$.)

The most interesting and nontrivial situation appears in the case when $\mu$ is imaginary, i.e. $b_{0} / C<0$, because as we shall see later, here the quantum central charge is less than $1, C_{q}<1$. We know degenerate representations are present. Here the effective action can be computed in the same way as in the previous case and it takes the form

$$
L_{\mathrm{eff}}=\frac{1}{8 \pi}\left[\left(\frac{C}{6}+2\right) \dot{\varphi} \varphi^{\prime}+2 i R \varphi\right] .
$$

It looks like (26), however $\varphi$ is complex, the first term has the opposite sign, and moreover there is an $i$ in the second term. From the condition $f^{\prime}=\exp i \varphi$ we get that under the reparemetrization $\varphi$ changes as $\delta \varphi=\varepsilon \varphi-i \varepsilon^{\prime}$, and therefore the energy-momentum tensor acquires the form

$$
T=\frac{1}{8 \pi}\left[\phi^{\prime 2}+2 i\left(\alpha-\frac{1}{\alpha}\right) \phi^{\prime \prime}\right],
$$


where $\alpha^{2}=-\left(\frac{C}{6}+2\right), \phi=\alpha \varphi$. For the central charge now we get

$$
C_{q}=1-6\left(\alpha-\frac{1}{\alpha}\right)^{2}
$$

and it means that $C_{q}<1$ (we assume that $\alpha$ is real).

Following our prescription, now we must assume that $\tilde{\mu}$ is a dynamical variable: $\tilde{\mu}=\tilde{\mu}(t)$ and we will consider the theory with Lagrangian (36) on the model space, instead of the coadjoint orbit. Thus the action acquires a form similar to (31):

$$
A_{\text {eff }}=-\frac{\alpha^{2}}{2} \int d t \tilde{\mu} \dot{\varphi}_{0}+A_{\text {eff }}(\tilde{\varphi}) .
$$

But now the story is different, due to the identification (23). It means that now the space of the zero modes is a torus $\mathbf{T}^{2}$, which is compact, and as a result we will get a discrete spectrum for the corresponding quantum mechanical system. Therefore we have a discrete spectrum of the representations. (Note that a similar phenomena should play an important role in the elliptic case for Liouville theory [9].)

In fact, it is possible to consistently quantize only the torus with the "integer" symplectic volume $V=2 \pi K ; K \in \mathbf{Z}$. Here we will use a generalized quantization scheme, which applies also for the rational $K ; K \in \mathbf{Q}$. For this it is necessary to assume that $\frac{\alpha^{2}}{2}$ is a rational number. Let us suppose that $\alpha^{2}=p / q$, where $p$ and $q$ are integers. Then the quantum central charge (38) becomes

$$
C_{q}=1-6 \frac{(p-q)^{2}}{p q}
$$

which coincides with the central charge for the Kac discrete series. The volume of the phase space is equal to $2 \pi \frac{p}{2 q}$ and we must make it an integer. The standard trick is to pass to the covering space, namely to the $2 q$-fold covering, i.e. we must impose the identifications

$$
\begin{aligned}
& \varphi_{0} \sim \varphi_{0}+2 \pi n \alpha, \quad n, m \in \mathbf{Z}, \\
& \tilde{\mu} \sim \tilde{\mu}+2 q m \alpha,
\end{aligned}
$$

instead of (23); here we redefine $\varphi_{0} \rightarrow \alpha \varphi_{0}, \tilde{\mu} \rightarrow \alpha \tilde{\mu}$, which is in agreement with the definition $\phi=\alpha \varphi$.

Now we must introduce the Hamiltonian. As in the previous case, $b_{0} / C>0$, the natural Hamiltonian is $H=\int T(\phi) d x$ [see (37)]. But now, by contrast with the finite-dimensional case, this Hamiltonian depends on the parameter $\tilde{\mu}$, which is subject to the identification (41), i.e. $H$ doesn't live on the torus: $H_{\tilde{\mu}+2 q m \alpha} \neq H_{\tilde{\mu}}$. So, we will integrate over $\tilde{\mu}$ from 0 to $\infty$, but the contributions of the points (i) $\tilde{\mu}=\tilde{\mu}_{0}$ $+2 q m \alpha$ and (ii) $\tilde{\mu}=-\tilde{\mu}+2 q m \alpha$, with some $m$, will be collected in a block. The first rule is a consequence of (41). The second comes from the following arguments: the starting action (17) is invariant under the reflection $\tilde{\mu}_{0} \mapsto-\tilde{\mu}_{0}$, thus we can consider 
only positive $\tilde{\mu}_{0}, \tilde{\mu}_{0} \geqq 0$; but there exists such an integer $m$ that $-\tilde{\mu}_{0}+2 q m \alpha \geqq 0$. Orbits, corresponding to $\tilde{\mu}$ from (i) and (ii) are different, but the boundary conditions on $f$ are the same and therefore we collect their contributions to one object, to the "Block." Then the resulting path integral is a sum over blocks. (Let us note once more that such construction is dictated by the symplectic geometry and the noninvariance of the Hamiltonian.)

Thus our prescription is as follows: we must integrate over $\tilde{\mu}$ from 0 to $\infty$ and then decompose this integral as

$$
\int_{0}^{\infty} d \tilde{\mu}(\ldots)=\int_{0}^{q \alpha} \sum_{\tilde{\mu}^{\prime} \sim \tilde{\mu}}(\ldots) d \tilde{\mu},
$$

where the sum is over the equivalence classes $\tilde{\mu}^{\prime} \sim \tilde{\mu}$, defined by (i) and (ii). We use the integration region $(0, q \alpha)$ instead of $(0,2 q \alpha)$ as a consequence of (ii). (Now it is clear that we need the $2 q$-fold covering of the torus both for even and odd $p$.)

Now, after this discussion, we can calculate the path integral over the Virasoro model space:

$$
I\left(\varphi_{0}^{\prime}, \varphi_{0}^{\prime \prime} \mid T\right)=\sum_{k} \sum_{n} \int_{\varphi_{0}^{\prime}+2 \pi n \alpha}^{\varphi_{0}^{\prime \prime}}\left[d \varphi_{0}\right][d \tilde{\mu}][d \tilde{\varphi}] \exp i\left\{A_{\text {eff }}+\int_{0}^{2 \pi T}\left(H-\frac{1}{24}\right) d t\right\} \chi_{k}(n) .
$$

Here the sum over $n$ guarantees the periodicity over $\varphi_{0}$ (see [2]) and we introduce a $Z_{2 q}$-character $\chi_{K}(n)$ in (43), which corresponds to the $2 q$-fold covering of the torus; $k=0, \ldots, 2 q-1$. We can use the approach of [2] and obtain:

$$
I\left(\varphi_{0}^{\prime}, \varphi_{0}^{\prime \prime} \mid T\right)=\sum_{k} \sum_{n} \int_{0}^{q \alpha} d \tilde{\mu} \exp \left(i \frac{\tilde{\mu}}{2}\left(\varphi_{0}^{\prime \prime}-\varphi_{0}^{\prime}+2 \pi n \alpha\right)\right) \exp \left(i 2 \pi n \frac{k}{2 q}\right) \tilde{I}\left(\tilde{\mu}^{\prime}, T\right),
$$

where

$$
\widetilde{I}\left(\tilde{\mu}^{\prime}, T\right)=\sum_{\tilde{\mu} \sim \mu^{\prime}} \int[d \tilde{\varphi}] \exp i\left\{A_{\mathrm{eff}}(\tilde{\varphi})+\int_{0}^{2 \pi T}\left(H-\frac{1}{24}\right) d t\right\} .
$$

Let us use the following parametrization for the integer $k$ in (44):

$$
k=s p-l q,
$$

where $k \in[0,2 q-1] ; s=1, \ldots, q$ and $s$ is present twice in the pair $(s, l)$ with odd and even $l$. Using the identity,

$$
\sum_{n \in \mathbf{Z}} \exp \left(2 \pi i n\left(\frac{1}{2} \tilde{\mu} \alpha+\frac{k}{2 q}\right)\right)=\sum_{n \in \mathbf{Z}} \delta\left(\frac{1}{2} \tilde{\mu} \alpha+\frac{k}{2 q}-n\right),
$$

we get for the possible values of $\tilde{\mu}$ :

$$
\tilde{\mu}=(2 n+l) \alpha^{-1}-s \alpha=r \alpha^{-1}-s \alpha,
$$

where $r$ is an integer; $r \in \mathbf{Z}$ and $s=1, \ldots, q$. Finally the path integral (44) acquires the form

$$
I\left(\varphi_{0}^{\prime}, \varphi_{0}^{\prime \prime} \mid T\right)=\sum_{r, s} \exp \frac{i}{2}\left(r \alpha^{-1}-s \alpha\right)\left(\varphi_{0}^{\prime \prime}-\varphi_{0}^{\prime}\right) \cdot \bar{\chi}_{r, s}(T)
$$

with

$$
s=1, \ldots, q ; \quad s \alpha^{2} \leqq r<(q+s) \alpha^{2},
$$


and

$$
\tilde{\chi}_{r, s}(T)=\sum_{\tilde{\mu}^{\prime} \sim\left(r \alpha^{-1}-s \alpha\right)} I\left(\tilde{\mu}^{\prime}, T\right)
$$

where $I\left(\tilde{\mu}^{\prime}, T\right)$ is given by (34). It is easy to show that

$$
\tilde{\chi}_{r, s}(T)=\frac{1}{\eta(T)} \sum_{n \in \mathbf{Z}} \exp 2 \pi i T \frac{\left(r \alpha^{-1}-s \alpha+2 q n \alpha\right)^{2}}{4} .
$$

Each block $\tilde{\chi}_{r, s}$ corresponds to the infinite set of orbits. The "Hilbert" space of this system is the set of the Verma modules with highest weights

$$
h_{n}=\frac{1}{4}\left(r \alpha^{-1}-s \alpha+2 q n \alpha\right)^{2} \text {. }
$$

The difference $h_{h}-h_{m}$ is an integer for any $m$ and $n$. The minimal value of $h$ from this set is

$$
h_{0, r, s}=\frac{(r q-s p)^{2}}{4 p q}
$$

(the choice of the minimal value of $h$ in this set corresponds to the choice of modules with nonzero cohomology in [12]) which coincides with the highest weight from the Virasoro discrete series (it differs from the standard Kac formula by the $\left.\frac{C_{q}-1}{24}\right)$, but the domain for the numbers $r$ and $s$ is different: instead of $1 \leqq s$ $\leqq q-1,1 \leqq r \leqq p-1, q r>p s$ in the Kac formula here we have $1 \leqq s \leqq q, s p / q \leqq r \leqq p$ $+s p / q$.

For each pair $(r, s)$ from the Kac spectrum there exist a pair $(\bar{r}, \bar{s})$ in $(49)$, defined by the following rule:

$$
\begin{gathered}
\bar{r}=2 p-r, \quad \bar{s}=s \quad \text { if } \quad r \alpha^{-1}+s \alpha \geqq q \alpha, \\
\bar{r}=p+r, \quad \bar{s}=q-s \quad \text { if } \quad r \alpha^{-1}+s \alpha \leqq q \alpha .
\end{gathered}
$$

The block $(\bar{r}, \bar{s})$ has beautiful properties: first $h_{0}^{(r, s)}-h_{0}^{(\bar{r}, \bar{s})}$ is an integer (it means that Verma moduli $V^{(\bar{r}, \bar{s})}$, corresponding to $h_{0}^{(\bar{r}, \bar{s})}$ is a submodule in $\left.V^{(r, s)}\right)$; second

$$
\chi_{r, s}(T)=\tilde{\chi}_{r, s}(T)-\tilde{\chi}_{r, s}(T)
$$

is a character of the degenerate Virasoro representation with highest weight $h_{0}^{r, s}$ and the central charge (40) from the Kac spectrum.

All this means that our theory is not "complete" (or the prescription is not complete), because in the "complete" theory the blocks must coincide with the characters of the degenerate representations; thus our blocks $\tilde{\chi}_{r, s}$ and $\tilde{\chi}_{\bar{r}, s}$ are from one "super block." It seems that the way to construct the "complete" theory is the following. We must consider quantum mechanics on the model space with wave functions which are not only functions but also differential forms. It is equivalent to a transition from the manifold to a supermanifold, and thus we must include the anticommuting variables into the path integral. Our hypothesis is that $\tilde{\chi}_{r, s}$ and $\tilde{\chi}_{\bar{r}, \bar{s}}$ will be from one supermultiplet and the path integral will reduce to the supertrace; as a result we must get the sum over blocks, where each block will be a character $\chi_{r, s}=\tilde{\chi}_{r, s}-\tilde{\chi}_{r, s^{*}}$. We hope to return to this question in a separate publication. 
At the end of this section we want to note that here we discover only the domain $C_{q}>25$ and $C_{q}<1$. The domain $1 \leqq C_{q} \leqq 25$ corresponds to the complex $\alpha$ in (30) and (37) and must be treated separately.

\section{Finite Sums in the RCFT; Kac-Moody Group}

In this section we discuss the quantization of the model space for the Kac-Moody group. The corresponding geometrical action is given by [3]

$$
S=\int \operatorname{Tr}\left\{-V_{0} g^{-1} d g-\frac{K}{4 \pi} g^{-1} d g g^{-1} \partial g+\frac{K}{4 \pi} d^{-1}\left(g^{-1} \partial g\left(g^{-1} d g\right)^{2}\right)\right\} d x
$$

and differs from the WZ action by the first term; here $\partial=\partial / \partial x ; g(x, t) \in L G$ (for concreteness we shall consider $G=S U(n)), V_{0}$ is a constant matrix from the Cartan subalgebra of the corresponding semi-simple Lie algebra, $K$ is a central charge of the Kac-Moody algebra. In the construction of the model space, following our prescription we must consider $V_{0}$ as a dynamical variable. As in the finite dimensional case we can assume that $V_{0}$ is a diagonal matrix from the positive Weyl chamber, i.e. its eigenvalues are $V_{0}^{1} \geqq \ldots \geqq V_{0}^{1}$. We'll discuss only the Hilbert space of the corresponding quantum-mechanical system, which is responsible for the quantization of the representations. We shall not introduce the Hamiltonian, and therefore we can consider the fundamental domain for $V_{0}$ with respect to the symmetries of the action.

Let us describe the symmetry group $\Gamma$ of the action $S$. First, there is a symmetry

$$
g \rightarrow g h_{D}
$$

where $h_{D}$ is a constant diagonal matrix (in general $h_{D}$ is from the maximal torus) $h_{D} V_{0} h_{D}^{-1}=V_{0}$, and therefore this transformation is not interesting for us. The discrete group $\Gamma / D$ contains the usual (finite) Weyl group

$$
g \rightarrow g w, \quad V_{0} \rightarrow w^{-1} V_{0} w, \quad w \in W,
$$

and the affine transformation

$$
g \rightarrow g e^{u_{0} x}, \quad V_{0} \rightarrow V_{0}+\frac{K}{2 \pi} u_{0},
$$

here $\exp 2 \pi u_{0}=1$. The last condition guarantees the conservation of the periodicity of $g(x)$ :

$$
g(2 \pi, t)=g(0, t) .
$$

It means that $u_{0}$ is from the root lattice of the corresponding semisimple Lie algebra. If $K=1$ the fundamental domain for the $2 \pi V_{0}$ coincides with the affine Weyl chamber:

$$
2 \pi\left\langle\alpha_{i}^{\vee}, V_{0}\right\rangle \geqq 0, \quad 2 \pi\left\langle\tilde{\alpha}^{\vee}, V_{0}\right\rangle \leqq 1
$$

where $\alpha_{i}$ are simple roots, $\tilde{\alpha}$ is the longest root, $\alpha^{\vee}=\frac{2 \alpha}{\langle\alpha, \alpha\rangle}$ dual root. For the $S U(n)$ case the simple roots are $\alpha_{i}=\operatorname{diag}\left(0, \ldots, 1_{(i)},-1_{(i+1)}, \ldots, 0\right)$, the longest root is $\tilde{\alpha}=(1,0, \ldots, 0,-1), \alpha^{\vee}=\alpha$ and pairing is given by trace. For the generic $K$ the 
fundamental domain is $K$ times the largest alcove

$$
2 \pi\left\langle\tilde{\alpha}^{V}, V_{0}\right\rangle \leqq K,
$$

and this alcove we define as $A_{K}$. The weights from $A_{K}$ correspond to (different) integrable representations.

Let us now introduce the dynamical variable $g_{0}$ conjugate to $V_{0}$ by $g=\tilde{g} g_{0}$, where $g_{0}=g_{0}(t)$ and

$$
\phi=\int \tilde{g}^{-1} \dot{\tilde{g}} d x=0 .
$$

In these variables we have

$$
S=\int \operatorname{Tr}\left\{-V_{0} g_{0}^{-1} d g_{0}-g_{0} V_{0} g_{0}^{-1} \tilde{g}^{-1} \dot{\tilde{g}}\right\} d x+W_{K}(\tilde{g}) .
$$

Here $W_{K}(\tilde{g})$ is the standard WZNW action. As it follows from this condition $\phi=0$, this action is factorized into two parts: the first one corresponding to the quantum mechanics and the second to the $2 d$ field theory. We are interested in the first term. Path integral now acquires the form

$$
I=\int\left[d V_{0}\right]\left[d g_{0}\right] \exp \left\{-i \int d t \operatorname{Tr}\left(2 \pi V_{0} g_{0}^{-1} \dot{g}_{0}\right)\right\} \cdot \int[d \tilde{g}] \exp \left(i W_{K}(\tilde{g})\right),
$$

where $V_{0} \in A_{K}$. The quantum mechanical part is easy to compute using the construction from Sect. 2,

$$
\operatorname{Tr} 2 \pi V_{0} g^{-1} d g=\sum_{i} \Delta_{i} d \varphi_{i}^{(0)}+\sum_{i, k} \Delta_{i}^{(k)} d \varphi_{i}^{(k)} .
$$

Path integral reduces to a sum over integer weights $\Delta^{\alpha}$ from the $A_{K}$, i.e. over the integrable representations of the Kac-Moody group,

$$
I=\sum_{\alpha} \exp \left(-i \sum_{i} \Delta_{i}^{\alpha}\left(\varphi_{i}^{0^{\prime \prime}}-\varphi_{i}^{0^{\prime}}\right)\right) \int d \Delta_{i}^{(k)} d \varphi_{i}^{(k)}[d \tilde{g}] \exp (i S(\Delta, \varphi, \tilde{g})),
$$

where $\varphi_{i}^{0^{\prime \prime}}$ and $\varphi_{i}^{0^{\prime}}$ are the boundary conditions.

Adding the Hamiltonian to the action (f.e. a linear sum of Cartan elements) we can define a character as a path integral with periodic boundary conditions. We think that using the construction, similar to those in the Virasoro case and the free field parametrization of the WZNW model (see f.e. [8]) it is possible to prove that the corresponding path integral over $g_{0}, V_{0}$, and $\tilde{g}$ is equal to sum of the Weyl-Kac characters over integrable representations.

\section{Appendix}

Here we'll discuss the relation between the geometrical action on the coadjoint orbit of the Virasoro group and classical $r$-matrix. We think that it will help us to understand the appearance of the quantum group in RCFT.

A similar relation can be found between the geometrical action, corresponding to the Kac-Moody and W-algebras (geometrical action here is given by the Drinfeld-Sokolov reduction from those for $S L(n)$ Kac-Moody group as in [3] for $S L(2)$, see f.e. the second ref. in [6]) and $r$-matrices in the Toda field theory.

Our main observation is that the geometrical action defines a non-degenerate symplectic structure on the loop group (cf. with [14]). It is easy to calculate the 
corresponding Poisson brackets for the Virasoro case from (19),

$$
\{f(x), f(y)\}=\alpha\left[\varepsilon(x-y)(f(x)-f(y))^{2}+f^{2}(x)-f^{2}(y)\right],
$$

where $\alpha=-48 \pi / C$ and $f(x)$ is a fundamental field with initial Boundary conditions in $2 d$ gravity. Now we'll demonstrate that these Poisson brackets are of the $r$-matrix nature. We mean here that the same Poisson brackets appeared earlier in the $r$-matrix approach for the Liouville-theory (see $[9,10,11])$.

Let us give a short summary of the r-matrix construction for Liouville theory following [9]. The fundamental relation which can be postulated is

$$
\{T(x) \otimes T(y)\}=-[r, T(x) \otimes T(y)],
$$

where $r \in \operatorname{sl}(2) \otimes \operatorname{sl}(2)$ is a classical constant $r$-matrix and $T$ is a $2 \times 2$ monodromy matrix. [The quantized version of this classical relation is the quantum group $\left.s l(2)_{q} \cdot\right] r$ is defined as a module, a multiple of the Casimir operator. The special choice of this multiple leads to the $r$-matrices

$$
\begin{gathered}
r^{+}=\frac{1}{4}\left[\sigma^{3} \otimes \sigma^{3}+2 \sigma^{+} \otimes \sigma^{-}\right], \\
r^{-}=-\frac{1}{4}\left[\sigma^{3} \otimes \sigma^{3}+2 \sigma^{-} \otimes \sigma^{+}\right],
\end{gathered}
$$

satisfying the classical Yang-Baxter equation. It follows from the relation $T(x)$ $=T(x, y) T(y)$ and the ultralocality condition (i.e. we suppose that $\{T(x) \otimes T(y, z)\}=0$ if $x \leqq y$ or $x \geqq z)$ that

$$
\{T(x) \otimes T(y)\}=T(x) \otimes T(y)\left[r-T^{-1}(y) \otimes T^{-1}(y) r T(y) \otimes T(y)\right]
$$

for $x>y$. These relations actually involve only two independent fields $u=B / A$ and $\mathscr{V}=D / C$ in the notation of [9], where we denote $T=\left(\begin{array}{ll}A & B \\ C & D\end{array}\right)$. For these fields we get from (69)

$$
\begin{gathered}
\{u(x), u(y)\}=\varepsilon(x-y)(u(x)-u(y))^{2}+u^{2}(x)-u^{2}(y) \\
\{\mathscr{V}(x), \mathscr{V}(y)\}=\varepsilon(x-y)(\mathscr{V}(x)-\mathscr{V}(y))^{2}+\mathscr{V}^{2}(x)-\mathscr{V}^{2}(y), \\
\{u(x), \mathscr{V}(y)\}=2\left(u(x) \mathscr{V}(y)-\mathscr{V}^{2}(y)\right) .
\end{gathered}
$$

Thus we see that the Poisson bracket for the "chiral" part, i.e. for $u(x)$ coincides with that for the fundamental field $f(x)$ in $2 d$ gravity. The absence of the second field $\mathscr{V}$ in the geometrical action is easy to understand: as we demonstrate above, quantization of the model space (or the orbit) corresponds to the chiral part of RCFT.

Acknowledgements. We would like to thank L. Faddeev, D. Yurev and especially M. SemenovTian-Shansky for stimulating discussions.

Note added. After the present paper was written, we received preprint [13] with results related to our Sect. 4. 


\section{References}

1. Alekseev, A., Shatashvili, S.: Propagator of relativistic spinning particle via path integral over trajectories. Mod. Phys. Lett. A 3, 1551-1559 (1988)

2. Alekseev, A., Faddeev, L., Shatashvili, S.: Quantization of symplectic orbits of compact Lie group by means of functional integral. J. Geom. Phys. (in press)

3. Alekseev, A., Shatashvili, S.: Path integral quantization of the coadjoint orbits of the Virasoro group and $2 d$ gravity, preprint LOMI-E-16-88 (1988)

4. Kirillov, A.A.: Elements of the theory of representations. Berlin, Heidelberg, New York: Springer 1979

5. Polyakov, A.M.: Quantum gravity in two dimensions. Mod. Phys. Lett. A 2, 893-898 (1987); Knizhnik, V., Polyakov, A., Zamolodchikov, A.: Fractal structure of $2-d$ quantum gravity. Mod. Phys. Lett. A 3, 819-829 (1988)

6. Belavin, A.A.: $\mathrm{KdV}$ equation and $W$-algebras, Lecture given at Taniguchi foundation symposium, Integrable models in quantum field theory and statistical Physics, Kyoto, 1988 Bershadsky, M., Ooguri, H.: Hidden $S L(n)$ symmetry in conformal field theories. Commun. Math. Phys. 126, 49-83 (1989)

7. Bernstein, J., Gelfand, I., Gelfand, S.: Proceedings of Petrovsky seminar, vol. 2, pp. 3-21 (1976) (in Russian)

8. Gezasimov, A., Marshakov, A., Morozov, A., Olshanetsky, M., Shatashvili, S.: Wess-Zumino model as a theory of free fields, I, II, III, IV, Preprints ITEP-64-89, ITEP-70-89, ITEP-72-89, ITEP-74-89 (1989)

9. Faddeev, L., Takhtajan, L.: Liouville model on the lattice. Lecture Notes in Physics, vol. 246, pp. 166-179. Berlin, Heidelberg, New York: Springer 1986

10. Gervais, J.L., Neveu, A.: New quantum treatment of Liouville field theory. Nucl. Phys. B 224, 329-348 (1983)

11. Babelon, O.: Etended conformal algebra and Yang-Baxter equation. Preprint PAR LPTHE $88 / 33,1988$

12. Felder, G.: BRST approach to minimal model. Nucl. Phys. B 317, 215-236 (1989)

13. Elitzur, S., Moore, G., Schwimmer, A., Seiberg, N.: Remarks on the canonical quantization of the Chern-Simons-Witten theory, Preprint IASSNS-HEP-89/20, 1989

14. Witten, E.: Coadjoint orbits of Virasoro group. Commun. Math. Phys. 114, 1-60 (1988)

Communicated by A. Jaffe

Received August 28, 1989 